\title{
ON THE UNIQUENESS OF COMPACT SOLUTIONS FOR CERTAIN ELLIPTIC DIFFERENTIAL EQUATIONS ${ }^{1}$
}

\section{ALFRED AEPPLI}

Summary. Let $O$ be a fixed point in $(n+1)$-space $R^{n+1}, r=r(p)$ the distance of $p$ from $O, p \in R^{n+1}, H_{\nu}$ the $\nu$ th mean curvature of an $n$-dimensional surface in $R^{n+1}$. In this paper it is proved that the only compact $n$-surfaces satisfying $r^{\nu} H_{\nu}=1$ are the spheres with center $O$. Generalizations of this proposition are given in b and c. §a contains two lemmas: Lemma 1 asserts the ellipticity of certain differential equations; in Lemma 2 a strong form of the "maximum principle" (due to E. Hopf) is stated for solutions of elliptic differential equations.

a. Let $F$ be an $n$-surface in $(n+1)$-space, $F^{n} \subset R^{n+1}, n \geqq 2, F$ of class $C^{2} .^{2} F$ has no singularities; self-intersections are allowed; $F$ is connected. The $\nu$ th mean curvature is the $\nu$ th elementary symmetric function of the principal curvatures $k_{1}, k_{2}, \cdots, k_{n}\left(k_{1} \geqq k_{2} \geqq \cdots \geqq k_{n}\right)$ divided by $C_{n, v}$ :

$$
\begin{aligned}
& H_{\nu}=C_{n, \nu}{ }^{-1} \sum k_{1} k_{2} \cdots k_{\nu}, \quad \nu=1,2, \cdots, n . \\
& H_{0}=1 .
\end{aligned}
$$

We use cartesian coordinates $x^{1}, x^{2}, \cdots, x^{n}, x^{n+1}=z$ in $R^{n+1}$ such that $F$ is given by $z=z\left(x^{1}, x^{2}, \cdots, x^{n}\right)$ in a neighborhood $U(p)$ of the point $p, p \in F, p=$ origin of the $(x, z)$-system. Then $H_{\nu}(\nu \geqq 1)$ is a differential expression of second order, i.e. an expression in $x=\left(x^{1}, x^{2}, \cdots, x^{n}\right), z, z_{i}=\partial z / \partial x^{i}=\partial_{i} z, z_{i j}=\partial_{i} \partial_{j} z(i, j=1,2, \cdots, n)$; $H_{\nu}$ is actually a polynomial in the $z_{i j}$ 's, and its coefficients are rational functions of the $z_{i}$ 's ( $x$ and $z$ do not occur). Let $r=r(q) \geqq 0$ be the distance of $q\left(q \in R^{n+1}\right)$ from the fixed point $O . r^{\nu} H_{\nu}$ is the $\nu$ th reduced mean curvature (cf. [3]). Let $c$ be a constant.

Lemma 1. (a) $H_{1}=c$ and $r H_{1}=c(r>0)$ are everywhere absolutely elliptic (partial) differential equations.

(b) If $k_{i}>0$ for all $i=1,2, \cdots, n$, then $H_{\nu}=c$ and $r^{\nu} H_{\nu}=c(r>0)$ are absolutely elliptic differential equations.

Received by the editors November 12, 1959.

1 The author was supported during the preparation of this paper by the Office of Naval Research under Contract No. Nonr 401(20)-NR043-167.

${ }^{2}$ In the sequel, $F$ will frequently be a solution of an absolutely elliptic partial differential equation of second order $\Phi=0$ (cf. [2, p. 107-113], for the definitions involved). If $F$ is of class $\mathrm{C}^{3}$ and if $\Phi$ is analytic, then $F$ is analytic by Bernstein's theorem. 
Proof. Let $x=\left(x^{1}, x^{2}, \cdots, x^{n}\right), x^{n+1}=z$ be coordinates as above, and let $F$ be in contact with the $x$-plane at $p$ (e.g. in positive contact: $\left.\mathfrak{n}(p)=\mathfrak{e}_{n+1}\right)$. We may assume that $x^{1}, x^{2}, \cdots, x^{n}$ are local coordinates in $U(p) \subset F$ with the property

$$
g_{i j}(p)=\delta_{i j}
$$

Therefore, we get from (cf. $[1, \S 1])$

$$
\begin{gathered}
\left|g^{i j} l_{j m}-k \delta_{m}^{i}\right|=0 \\
\text { (eigenvalues } k=k_{1}, k_{2}, \cdots, k_{n} ; i, j, m=1,2, \cdots, n \text { ), }
\end{gathered}
$$

together with $l_{j m}=\mathfrak{x}_{j m} \mathfrak{n}$, the relation

$$
\left|\left(\delta^{i j}+\Delta^{i j}(x)\right) \cdot z_{j m} \cdot\left(1+\Lambda_{j m}(x)\right)-k \delta_{m}^{i}\right|=0,
$$

where $\Delta^{i j}(p)=0$ and $\Lambda_{j m}(p)=0$. (2) implies (according to (1))

$$
\begin{aligned}
& C_{n, \nu} H_{\nu}(x)=\sum z_{11} z_{22} \cdots z_{\nu \nu}+P_{\nu}\left(x, z, z_{i}, z_{i j}\right), \\
& P_{\nu} \text { polynomial in the } z_{i j} \text { 's, whose coefficients vanish for } x=p .
\end{aligned}
$$

Using an appropriate numeration of the coordinates $x^{i}$, (2) yields

$$
z_{j j}(x)=k_{j}(p)+Q_{j}(x), \quad Q_{j}(p)=0 .
$$

Let $\left(H_{\nu}\right)_{(i j)}=\partial H_{\nu} / \partial z_{i j}$. (3) and (4) imply

$$
C_{n, \nu}\left(H_{\nu}\right)_{(i j)}(p)=\delta_{i j} \cdot\left(\sum^{(j)} k_{1} \cdots \hat{k}_{j} \cdots k_{v}\right)(p)=C_{n, \nu} \delta_{i j} \frac{\partial H_{\nu}}{\partial k_{j}}(p),
$$

where $\hat{k}_{j}$ means replacement of $k_{j}$ by 1 , and $\sum^{(j)}$ summation over all summands $k_{1} k_{2} \cdots k_{\nu}$ in (1), in which $k_{j}$ occurs. We get from (5)

$$
\left.\begin{array}{c}
\sum_{i \leq j}\left(H_{\nu}\right)_{(i j)} Y^{i} Y^{j} \text { positive definite } \\
\sum_{i \leq j}\left(r^{\nu} H_{\nu}\right)_{(i j)} Y^{i} Y^{j} \text { positive definite }
\end{array}\right\} \begin{array}{r}
\text { for } \nu=1, \text { and for } \nu=2,3, \cdots, n \\
\text { if } k_{i}>0(\text { for all } i ; r>0) .
\end{array}
$$

This proves (a) and (b).

Lemma 2. Let $F_{1}, F_{2}$ be two (regular) surfaces in contact at $p$ both of which are solutions of the absolutely elliptic (partial) differential equation (of second order) $\Phi=0\left(F_{1}, F_{2}\right.$ of class $C^{2} ; \Phi=\Phi\left(x, z, z_{i}, z_{i j}\right)$ of class $C^{0}$ in all variables $x^{1}, x^{2}, \cdots, z_{n n}$ and of class $C^{1}$ in $z, z_{1}, z_{2}, \cdots$, $\left.z_{n n}\right)$. Then the intersection of $F_{1}$ and $F_{2}$ consists of a set ${ }^{3} N(p \in N)$ such

${ }^{3}$ In the analytic case: $N$ is an $(n-1)$-surface which is in the first approximation a cone with vertex $O ; N$ may have several locally irreducible components, for $n=2$ there must be at least two of them. 
that the contact at $p$ between $F_{1}$ and $F_{2}$ is not semi-proper, ${ }^{4}$ or else $F_{1}$ and $F_{2}$ coincide in a neighborhood of $p$.

Proof following [2], with the help of [4] and [5]: the difference of two solutions of $\Phi=0$ solves a homogeneous linear elliptic differential equation (cf. [2, p. 109]); application of [4, Satz 3, p. 149] and of [5] leads immediately to Lemma 2 .

b. Let $S_{o}$ be the $n$-sphere in $R^{n+1}$ with center $O$ (and with any (positive) radius).

\section{Proposition 1. Let $F$ be compact, and let}

$$
r^{\nu} H_{\nu}=1
$$

on $F$ (for a fixed $\nu ; 1 \leqq \nu \leqq n)$. Then $F=S_{0}$.

Proof. Since $F$ is compact, there exists a sphere $S_{o}$ which touches $F$ "from outside": take a sphere with center $O$ and a large radius such that the intersection with $F$ is empty, and shrink this sphere homothetically until it touches $F$ for the first time. In the neighborhood of a point of contact we take the inner normals as positive (the inner normal is the positive one on $S_{o}$ globally; for odd $\nu$ we have to make this choice because (6) holds for $S_{o}$ and $F$ ). Then the contact is positive, and the principal curvatures of $F$ (and of $S_{o}$ ) are all positive at the points of contact. Lemma 1 for $r^{\nu} H_{\nu}=1$ and Lemma 2 yield coincidence of $F$ with $S_{o}$ in a neighborhood of a point of contact, i.e. the set of points of contact is open. By continuity it is also closed, therefore $F=S_{o}$ (since $F$ is compact and connected).

Remark. In the proofs of Proposition 1 and later propositions we use only Satz 3 of [4] (maximum principle for linear elliptic differential equations in an open domain), and not the full strength of Lemma 2 (i.e. of [5]) with the notion of semi-proper contact. However, Lemma 2 is useful in the case of surfaces with boundary. We get theorems (with proofs analogous to the one above) similar to Satz $1^{\prime}$ and Satz $10^{\prime}$ in [3] for a central projection $T: F \rightarrow \bar{F}$ between two surfaces $F, \bar{F}$ with regular boundary and with $O$ as center of the projection $T, O \notin F$ (and therefore $O \notin \bar{F}$ too). We see: all the propositions in this paper possess their counterpart theorems (similar to Satz $1^{\prime}$ in [3]) about central projections between surfaces with regular boundary. Parallel projections can be treated the same way (cf. [1]).

- If $F_{1}$ and $F_{2}$ have a contact at $p$ such that there is on $F_{1}$ a regular $(n-1)$-surface $A$ through $p$ with the property that in a neighborhood of $p$ on at least one side of $A, F_{1}$ and $F_{2}$ do not intersect, then $F_{1}$ and $F_{2}$ have a semi-proper contact at $p$ (cf. $[2$, p. 102]; "intersection" in the strong sense of 3 in $[2$, p. 102]).

- $F$ of class $C^{2}$, as always. 
We assume in the following propositions $1^{\prime}$ and $1^{\prime \prime}$ that $F$ is compact and without self-intersections $\left(R^{n+1}-F=I \cup E\right.$ with $I \cap E$ empty, $I$ is the interior, $E$ the exterior of $F, E$ containing the point at $\infty$ ), that $O \in I$, and that the positive normal $\mathfrak{n}_{+}$is always the inner normal. Furthermore, let the occurring differential equations be taken with respect to this choice of $\mathfrak{n}_{+}$.

Proposition 1'. Let

$$
\sum_{\nu=1}^{n} a_{\nu} r^{\nu} H_{\nu}=\sum_{\nu=1}^{n} a_{\nu}>0
$$

on $F$ with nonnegative functions $a_{\nu}$ (defined and of class $C^{1}$ on $\left.R^{n+1}-O\right) .^{6}$ Then $F=S_{0}$.

The proof is similar to that of Proposition 1, since $\left(6^{\prime}\right)$ is absolutely elliptic for $k_{i}>0(i=1,2, \cdots, n), a_{\nu} \geqq 0, \sum a_{\nu}>0$.

An analogous argument shows:

Proposition $1^{\prime \prime}$. Let $\Phi=0$ be an absolutely elliptic differential equation for $k_{i}>0(i=1,2, \cdots, n)^{7}$ such that every sphere $S_{o}$ is a solution of $\Phi=0$. Then every solution $F$ (with the properties mentioned above) is a sphere $S_{0}$.

Remark. Proposition 1 was proved in [3] for $\nu=1, F \in \Omega(S, O)$ (i.e. $F$ "star-shaped" in the sense of $[3, \S 3])$; furthermore for $\nu$ $=2,3, \cdots, n, F \in \mathfrak{R}(S, O)$. The proofs were based on the following relationships ((3.6) and (6.6) in [3]; $S=n$-sphere in $R^{n+1}$ with center $O$ and radius $1 ; \kappa_{n}=$ surface area of $\left.S\right)$ :

$$
\begin{array}{cc}
\int_{S} r H_{1} d \Omega \leqq \kappa_{n} & \text { for } F \in \Re(S, O), \quad “={ } \Leftrightarrow F=S_{o}, \\
\int_{S}{ }^{\nu} H_{\nu} d \Omega \leqq \kappa_{n} & \text { for } F \in \mathfrak{R}(S, O), \quad \text { “= } \Leftrightarrow F=S_{o}, \\
\nu=2,3, \cdots, n .^{8}
\end{array}
$$

c. Let $f(q)$ be a function of class $C^{1}$ on $S$ ( $q \in S, S$ as above), and let [ $p q]\left(p, q \in R^{n+1}, p \neq q\right)$ be the straight line in $R^{n+1}$ through $p$ and $q$. Let the compact surface $G$ have no self-intersections, let $\mathfrak{n}_{+}$be the inner normal, and let $O \in I$ ( $I=$ interior of $G)$.

- $a_{\nu}$ may even depend on the normal of $F$. Then $a_{\nu}$ is supposed to be a function of class $C^{1}$ on the tangent bundle of $R^{n+1}-O$ (i.e. on the manifold of the elements $(b, p), b$ unit vector in $R^{n+1}, \mathrm{p} \in R^{n+1}-O$ ).

7 Continuity and differentiability of $\Phi$ as in Lemma 2 .

8 I do not know whether $\left(7_{v}\right)$ is true for $F \in \Omega(S, O)$. 
Proposition 2. Assume

$$
r(p) H_{1}(p)=f(q) \quad \text { for } p \in F, q \in S, p \in[O q],
$$

has a solution ${ }^{5} F$ which is star-shaped ${ }^{9}$ with respect to $O$ (and therefore all $F_{t}$ are solutions, where $F_{t}$ and $F$ are homothetic with the factor $t>0$ and with respect to $O$ ). Then every solution $G$ (of class $C^{2}$ and with the properties mentioned above) is such an $F_{t}$.

The proof follows the same lines as that of Proposition 1: to every $G$ there exists an $F_{t}$ which is in positive contact with $G$ from outside, and therefore $G=F_{t}$ (for some $t$ ) as above. Proposition 2 generalizes Satz 1 in [3] (proved by help of an integral formula) for a starshaped $F$.

We may weaken the hypotheses in Proposition 2 because of the following fact: if $F$ is compact, without self-intersections, with $O \in I$, and if $F$ is a solution of (8), then $F$ is star-shaped. To see this, we consider $F^{\prime}=F_{t}$ for a sufficiently large $t>0$ such that $F \cap F^{\prime}$ is empty; then we contract $F^{\prime}$ again to a surface $F^{\prime \prime}$ with the property that $F^{\prime \prime}$ touches $F$ for the first time $\left(F^{\prime \prime}\right.$ is homothetic to $F$, and $F^{\prime \prime}$ touches $F$ from outside). The contact between $F$ and $F^{\prime \prime}$ is positive, since $O \in I$ (taking for $\mathfrak{n}_{+}$the inner normal). (8) implies $F=F^{\prime \prime}$ by help of the Lemmas 1 and 2 (and because $F$ is compact and connected), and $F$ has to be star-shaped with respect to $O$, i.e. every half-line starting at $O$ intersects $F$ in exactly one point, otherwise $F$ would have at least two (homothetic) components.

We get easily another proposition which is similar to Proposition 1:

Proposition 2 . Let an ovaloid $F$ with $O \in I$ be a solution of

$$
r^{\nu}(p) H_{\nu}(p)=f(q)>0, \quad p \in F, q \in S, p \in[O q],
$$

(and therefore all $F_{t}$ are solutions, $t>0$ ). $\nu$ is fixed, $1 \leqq \nu \leqq n$. Then every compact solution is such an $F_{t}$.

A priori, the solution of $\left(8_{v}\right)$ is here allowed to have self-intersections. The proof is again almost the same as above, the surface $F_{t}$ replacing the spheres $S_{0}$.

For $\nu=n$ every compact solution is automatically an ovaloid (Hadamard) with $O \in I(r>0$ because $f>0)$. Therefore, Proposition $2_{n}$ remains valid if we replace the condition for $F$ to be an ovaloid

${ }^{9}$ Here and in the sequel, we mean by " $F$ is star-shaped with respect to $O$ " the property that every half-line starting at $O$ intersects $F$ in exactly one point. "Starshaped" in the sense " $F \in \Re(S, O)$," as used in [3], is a slightly stronger condition. The differential equation ( 8 ) is taken with $\mathfrak{n}_{+}=$inner normal. 
by the compactness of $F$. Proposition 2 contains Satz 10 in [3], under the additional assumption $O \in I$. Thus Satz 10 (without the hypothesis (c) on the connecting family $\mathfrak{F}$ and leaving out the convexity condition in (b), cf. [3, p. 192]) can be proved for $O \notin F$ using the method explained here: since $\bar{F}$ (in Satz 10) is an ovaloid, the shadow frontier is a regular surface (if not empty); then we apply a proposition, analogous to Proposition $2_{\nu}$, for a central projection (with center $O$ ) between two surfaces $F$ and $\bar{F}$ with regular boundary $(O \notin F$; cf. remark after the proof of Proposition 1).

Let $G$ (of class $C^{2}$ ) in the Propositions $2^{\prime}$ and $2^{\prime \prime}$ be again compact, without self-intersections, let $O \in I$, and let $\mathfrak{n}_{+}$be the inner normal (the differential equations always being taken with $\mathfrak{n}_{+}=$inner normal).

Proposition 2'. Let an ovaloid $F$ with $O \in I$ be a solution of

$$
\sum_{\nu=1}^{n} a_{\nu} \nu^{\nu}(p) H_{\nu}(p)=f(q)>0, \quad p \in F, q \in S, p \in[O q],
$$

with $a_{\nu}=a_{\nu}(q) \geqq 0^{10}$ (and therefore all $F_{t}$ are solutions, $t>0$ ). Then every solution $G$ is such an $F_{t}$.

Proposition $2^{\prime \prime}$. Let $\Phi=0$ be an absolutely elliptic differential equation $^{7}$ with a one-parameter family of compact solutions $F_{t}, t>0, F_{t}$ homothetic to $F=F_{1}$ with the factor $t$ and with respect to $O, F$ without self-intersections, $O \in I$ (hence through any point $p \in R^{n+1}, p \neq O$, there passes at least one $\left.F_{t}\right)$. Then the surfaces $F_{t}$ are star-shaped with respect to $O$, and every solution $G$ is such an $F_{t}$.

The proofs are similar to the proofs of the foregoing propositions.

More generally, we can say: let the absolutely elliptic differential equation $\Phi=0$ have a one-parameter family of compact solutions $F_{t}, t>0, F_{t}$ continuously dependent on $t, F_{t} \rightarrow O$ for $t \rightarrow 0, d\left(O, F_{t}\right) \rightarrow \infty$ for $t \rightarrow \infty(d(X, Y)$ is the distance between $X$ and $Y)$, and all $F_{t}$ are supposed to be without self-intersections, $O \in I_{t}$ for all $t>0\left(I_{t}=\right.$ interior of $\left.F_{t}\right)$; the positive normal is taken as above; then every solution $G$ (with the properties given above) is such an $F_{t}$.

Added in proof. Since submitting this paper, I discovered that A. D. Alexandrov got theorems similar to the propositions above in Uniqueness theorems for surfaces in the large, Vestnik Leningrad University, 1958, no. 7, pp. 14-26 (Russian).

${ }^{10} a_{\nu}$ of class $C^{1} . a_{\nu}$ may depend on the normal of $F$. Then we assume that $a_{\nu}$ is a function of class $C^{1}$ on the tangent bundle of $R^{n+1} \mid S$ (extended homothetically to the tangent bundle of $R^{n+1}-O$ ). The same remark applies to $f$ (also in the previous propositions). 


\section{REFERENCES}

1. K. Voss, Einige differentialgeometrische Kongruenzsätze für geschlossene Flächen und Hyperflächen, Math. Ann. vol. 131 (1956) pp. 180-218.

2. H. Hopf, Lectures on differential geometry in the large, Stanford University, 1956.

3. A. Aeppli, Einige Aehnlichkeits- und Symmetriesätze für differenzierbare Flächen im Raum, Comment. Math. Helv. vol. 33 (1959) pp. 174-195.

4. E. Hopf, Elementare Bemerkungen über die Lösungen partieller Differentialgleichungen zweiter Ordnung vom elliptischen Typus, Sitzungsberichte der Berliner Akademie der Wissenschaften vol. 19 (1927) pp. 147-152.

5. - A remark on linear elliptic differential equations of second order, Proc. Amer. Math. Soc. vol. 3 (1952) pp. 791-793.

CORNELl University

\section{SOME RESULTS ON TAME DISKS AND SPHERES IN $E^{3}$}

\section{P. H. DOYLE AND J. G. HOCKING}

It is well known that there are cells and spheres of dimensions one and two in Euclidean 3-space $E^{3}$ which fail to be locally tame at a single point. Artin and Fox [1] have given many examples. Several authors have given characterizations, such as $[11 ; 12$ and 13$]$, of the tame cells among the "almost polyhedral" cells. The results in this paper are of the same general nature.

We use the term "disk" to mean a topological closed 2-cell. If $D$ is a disk, then $D^{0}$ denotes the interior of $D$ and $B d D$ denotes the boundary of $D$. The same notation also will be applied to an arc (a topological closed 1-cell).

The Theorem 0 below is stated without proof here, since a complete proof appears in Lemma 5.1 of [7]. We have used this result extensively and have noticed many modifications of it in the literature. It therefore seems useful to state it explicitly.

THEOREM 0. Let $D$ be a disk in $E^{3}$ which is locally polyhedral except at points of $B d D$. If $U$ is any open set containing $D^{0}$, then there is a 3-cell $C^{3}$ in $U \cup B d D$ such that (i) $D \subset C^{3}$, (ii) $D$ spans $B d C^{3}$, (iii) and $C^{3}$ is locally polyhedral except on $B d D$.

TheOREm 1. Let $D$ be a disk in $E^{3}$ and let $A$ be a tame arc on $D$ spanning BdD. If $D$ is locally polyhedral except on $A$, then $D$ is tame.

Proof. A remark will suffice to show that $D$ is locally tame at each point of $D^{0}$. In [4] it is shown that each point $x$ in $A^{0}$ lies in the

Received by the editors November 17, 1959. 